\title{
Utilização de biomarcadores cardíacos na detecção de infarto agudo do miocárdio
}

\author{
Use of cardiac biomarkers in the detection of acute myocardial infarction \\ Utilización de biomarcadores cardiacos en la detección de infarto agudo del miocardio \\ Lysandro Pinto Borges $^{1 *}$, Rafaella Campos Silva de Jesus ${ }^{1}$, Raissa Litsas Moura ${ }^{1}$.
}

\begin{abstract}
RESUMO
Objetivo: Analisar os biomarcadores cardíacos mais efetivos e solicitados para o diagnóstico do IAM. Métodos: Trata-se de revisão bibliográfica, que utilizou artigos e pesquisas científicas constantes em banco de dados como Sciello, Lilacs e outras bibliotecas virtuais. Os critérios de inclusão adotados foram: publicações cujo acesso fosse do texto na íntegra; que o resumo mantivesse relação com o objetivo proposto; trabalhos em todas as línguas; e, cujo ano de publicação não data mais de 12 anos. Resultados: Das 15 publicações utilizadas, 12 são revisão de literatura, 1 Estudo de caso, 1 Ensaio Clínico e 1 estudo transversal. As publicações utilizadas concluíram pelo uso de biomarcadores como prática eficiente no diagnóstico e prognóstico de doenças cardiovasculares. Conclusão: Apresentada as funções, e levando em consideração aspectos como especificidade, sensibilidade e perfil de liberação na corrente sanguínea, conclui-se que a Mioglobina e a fração MB da Creatinoquinase (CK-MB) possuem baixa especificidade por estarem presentes tanto na musculatura esquelética como também na musculatura cardíaca, mas são considerados sensíveis. Diferentemente das Troponinas cardíacas $\mathrm{T}$ e I, que mostram ter maior especificidade para o coração e sensibilidade clínica alta.
\end{abstract}

Palavras-chave: Marcadores Cardíacos, IAM, Troponinas, Mioglobinas, CK-MB.

\begin{abstract}
Objective: To analyze the most effective and requested cardiac biomarkers for the diagnosis of AMI. Methods: This is a bibliographical review, which used articles and scientific research in a database such as Scielo, Lilacs and other virtual libraries. The inclusion criteria adopted were publications that had access to the text in its entirety; that the abstract is related to the proposed objective; publications in all languages; and whose year of publication does not exceed 12 years. Results: Of the 15 publications used, 12 are literature review, 1 case study, 1 clinical trial and 1 cross-sectional study. The publications used concluded the use of biomarkers as an efficient practice in the diagnosis and prognosis of cardiovascular diseases. Conclusion: Given the specificity, sensitivity and profile of release in the bloodstream, it was concluded that Myoglobin and the MB fraction of creatine kinase (CK-MB) have low specificity for are present in both the skeletal musculature and cardiac musculature but are considered sensitive. Differently from cardiac and I cardiac troponins, which show greater specificity for the heart and high clinical sensitivity.
\end{abstract}

Key words: Cardiac markers, Acute myocardial infarction, Troponin, Myoglobin, Creatine kinase.

1Universidade Federal de Sergipe (UFS), Aracaju-Sergipe. *E-mail: lysandro.borges@gmail.com

SUBMETIDO EM: 5/2019

ACEITO EM: 6/2019

PUBLICADO EM: 7/2019

REAS/EJCH | Vol. 11(13) | e940 | DOI: https://doi.org/10.25248/reas.e940.2019 Página 1 de 8 


\section{RESUMEN}

Objetivo: Analizar los biomarcadores cardíacos más efectivos y solicitados para el diagnóstico del IAM. Métodos: Se trata de revisión bibliográfica, que utilizaron artículos e investigaciones científicas constantes en base de datos como Scielo, Lilacs y otras bibliotecas virtuales. Los criterios de inclusión adoptados fueron: publicaciones cuyo acceso fuera del texto en su totalidad; que el resumen mantuviera relación con el objetivo propuesto; publicaciones en todas las lenguas; y cuyo año de publicación no data más de 12 años. Resultados: De las 15 publicaciones utilizadas, 12 son revisión de literatura, 1 Estudio de caso, 1 Ensayo Clínico y 1 estudio transversal. Las publicaciones utilizadas concluyeron por el uso de biomarcadores como práctica eficiente en el diagnóstico y pronóstico de enfermedades cardiovasculares. Conclusión: Se ha demostrado que la Mioglobina y la fracción MB de la Creatinoquinasa (CK-MB) poseen una baja especificidad por estar presentes tanto en la musculatura esquelética como también en la musculatura esquelética y también en la sensibilidad y perfil de liberación en el torrente sanguíneo, en la musculatura cardiaca, pero se consideran sensibles. A diferencia de las Troponinas cardiacas T e I, que muestran tener mayor especificidad para el corazón y la sensibilidad clínica alta.

Palabras clave: Marcadores cardiacos, IAM, troponinas, mioglobina, CK-MB.

\section{INTRODUÇÃO}

Com o advento da modernização social, desenvolvimento tecnológico (principalmente nas áreas sanitárias e científicas), melhores condições de vida e acesso amplo a medicamentos, o envelhecimento da população é uma consequência natural. A expectativa de vida de 1940 a 2018 aumentou de 42,7 para 76 anos. Contudo, nas últimas décadas, mesmo com a maximização da qualidade de vida, registrou-se maior incidência de doenças crônico-degenerativas, que surgem mais na idade adulta, entre as quais pode se mencionar as doenças cardiovasculares (DCVs) (CANTELLE CF e LANARO R, 2011).

Atualmente as doenças coronarianas geram, aos cofres públicos, custos vultosos com internações hospitalares e tratamento todos os anos. De acordo com Organização Mundial de Saúde (OMS), o índice de mortalidade relacionado com DCVs, em 2012, foi de 214 para 100.000 habitantes brasileiros.

Destarte, elas representam $29 \%$ dos óbitos brasileiros, matando 2 vezes mais que todos os tipos de câncer, 2,5 vezes mais que todos os acidentes e mortes resultantes de violência e 6 vezes mais que as infecções, incluídas as mortes por AIDS (SBC, 2014; CARDOSO MR, et al., 2018).

A maioria dos casos de óbitos registrados são considerados evitáveis e não aconteceriam se os pacientes seguissem à prescrição dos medicamentos indicados pelos cardiologistas e realizassem exames preventivos. As principais DCVs que acometem homens e mulheres são: as Síndromes Coronarianas Agudas (Infarto Agudo do Miocárdio - IAM e Angina) e Insuficiência Cardíaca Congestiva (ICC). Um fator importante para prevenção destas doenças é o controle dos fatores de risco, entre os quais se podem citar o tabagismo, obesidade, sedentarismo, hipertensão arterial e alto nível de colesterol (SILVA SH e MORESCO RN, 2011; CANTELLE CF e LANARO R, 2011; SBC, 2014).

Vale dizer que o IAM é uma das principais causas de morte não só no Brasil, como no mundo, observandose um índice de aproximadamente 300 mil mortes anuais. $O$ infarto ocorre quando há evidência de necrose miocárdica em um contexto que se correlaciona com isquemia miocárdica aguda (LOZOVOY MAB, et al., 2008; CANTELLE CF e LANARO R, 2011; SOUZA LP, et al., 2016; CARDOSO MR, et al., 2018).

Em razão de sua alta taxa de morbimortalidade, a avaliação clínica precisa do IAM é de suma importância. Um dos exames mais utilizados é o eletrocardiograma (ECG). Entretanto, algumas alterações não são registradas por este exame, dificultando, assim, a sua identificação, daí ser essencial a determinação dos biomarcadores cardíacos, uma vez que eles tanto auxiliam no rastreamento da doença e estratificação de riscos, quanto contribuem para maior rapidez e precisão do diagnóstico e possibilitam intervenções que aumentem a sobrevida do paciente (SBC, 2014; MIRANDA MR e LIMA LM, 2014; SOUZA LP, et al., 2016). 
Neste contexto, fica evidente a importância destes recursos como prática clínica amplamente utilizada para identificação de DCVs, principalmente de IAM. Em razão disso, propõe-se como objetivo desta pesquisa a análise dos biomarcadores cardíacos mais efetivos e solicitados para o diagnóstico do IAM, de acordo com as literaturas estudadas relatando suas funções, grau de especificidade, sensibilidade e perfil de liberação na corrente sanguínea.

\section{MÉTODOS}

Trata-se de revisão bibliográfica, onde foram utilizados artigos e pesquisas científicas constantes em banco de dados como Scielo e Lilacs, e bibliotecas virtuais como da Universidade Federal de Sergipe. O levantamento biográfico foi realizado de outubro/2018 a fevereiro/2019, adotando-se como descritores os termos: Infarto do Miocárdio; Biomarcadores; Marcadores Cardíacos; Doenças Cardiovasculares; Troponina; Mioglobina; CK-MB.

Os critérios de inclusão adotados foram: publicações cujo acesso fosse do texto na íntegra; que o resumo mantivesse relação com o objetivo proposto; e, cujo ano de publicação não data mais de 12 anos. Seguindose tais critérios, das 20 publicações selecionadas, somente 15 foram utilizadas no desenvolvimento desta pesquisa.

Vale mencionar que este estudo apresenta o mínimo de risco, uma vez que se trata de revisão de literatura fundamentada em base de dados confiáveis, os benefícios são inúmeros, já que a mesma representa maior informação sobre o tema para população e para profissionais da área, podendo, inclusive, ser base para outras pesquisas científicas.

\section{RESULTADOS E DISCUSSÃO}

Para compor este estudo foram utilizadas 15 publicações que atendiam aos critérios previamente determinados, todas elas datando menos de 12 anos de publicado e apresentando relação direta com o tema e objetivo proposto. Das 15 publicações, 12 são revisão de literatura, 1 Estudo de caso, 1 Ensaio Clínico e 1 Estudo transversal.

Todos os artigos e dissertações de mestrado utilizadas concluíram pelo uso de marcadores biológicos como prática eficiente no diagnóstico e prognóstico de doenças cardiovasculares (Tabela 1, pág. 4).

A mioglobina é uma proteína globular com baixo peso molecular, presente tanto no músculo cardíaco quanto no esquelético. Embora ela não esteja presente no sangue, se conecta ao oxigênio mais fortemente que a hemoglobina funcionando como grande reservatório de $\mathrm{O} 2$ nos tecidos. Ela está presente em todas as fibras musculares estriadas (CANTELLE CF e LANARO R, 2011; SILVA SH e MORESCO RN, 2011).

Essa proteína é considerada um marcador precoce de lesão miocárdica, vez que ela é liberada uma a três horas após a morte da célula miocárdica, atingindo picos em 5 a 12 horas.

Além disso, esta enzima pode ser liberada sob diversas condições que não miocárdica (arritmias, distrofia muscular, choque, trauma, etc.), sendo sua vantagem a detecção do IAM nas primeiras horas de sua ocorrência (SBC, 2014; SOUZA LP, et al., 2016).

Existem vários testes para que a mioglobina seja dosada e, embora nenhum deles seja, de fato, satisfatório em razão da baixa especificidade cardíaca, deve ser retirada uma medida isolada desta enzima (4 a 8 horas) logo depois do primeiro desconforto no peito com Eletrocardiograma sem diagnóstico de IAM.

Esta concentração é determinada através de um teste turbidimétrico que avalia o ponto final de sua concentração, utilizando uma medição fotométrica da reação antígeno-anticorpo.

Entretanto, no caso de diagnóstico ainda impreciso, deve ser realizado complemento através da análise de CK-MB e Troponina I (Tnl) ou a Troponina T (TnT) (LOZOVOY MAB, et al., 2008; CANTELLE CF e LANARO R, 2011). 
Tabela 1 - Publicações utilizadas para composição da discussão.

Autor (es) Ano de Publicação

\section{Palavras Chaves}

Infarto agudo do miocárdio, perfil

LOZOVOY MAB, et al.

2008

enzimático, enzimas cardíacas, diagnóstico

Revisão de Literatura

MARTINS CS.

2009

CANTELLECF e

LANARO R.

2011

SILVA SH e

MORESCO RN.

ROCHA K.

2012

KATRUKHA IA.

2013

OYAMA MA.

2013 troponina cardíaca; isquemia; hipóxia Literatura

marcadores cardíacos, indicadores Revisão de bioquímicos, infarto agudo do miocárdio Literatura

doença cardíaca coronária; infarto do Revisão de miocárdio; marcadores biológicos. Literatura

Marcadores Bioquímicos; Infarto Agudo Revisão de do Miocárdio

Literatura

complexo troponina, contração muscular, fosforilação, troponina I, troponina $\mathrm{T}$, troponina $\mathrm{C}$

Revisão de

Literatura

BNP; Biomarcadores

cardíacos; Doença cardíaca; Peptídeo Ensaios clínicos natriurético; Troponina
Revisão de

\section{Principais Conclusões}

O IAM é sem dúvida a mais importante cardiopatia isquêmica que existe no mundo. Ela acomete pessoas de várias idades, porém com maior incidência na faixa acima dos 30 anos. O diagnóstico rápido é de fundamental importância para o prognóstico do paciente e eficácia do tratamento. O diagnóstico laboratorial é realizado pela determinação dos níveis séricos de proteínas e enzimas que extravasam, após o infarto

As troponinas cardíacas, e em especial a troponina I, são proteínas que funcionam como marcadores de lesão miocárdica, sendo altamente especiais e seletivas

Os marcadores cardíacos são importantes para diagnosticar infarto agudo do miocárdio, mas existem aqueles que são mais sensíveis e específicos para tal diagnóstico.

Vários marcadores têm apresentado eficácia na detecção da necrose, isquemia, inflamação, desestabilização da placa e disfunção cardíaca. No entanto, as troponinas cardíacas continuam sendo consideradas o padrão ouro para o diagnóstico do infarto agudo do miocárdio

Dos marcadores utilizados no diagnóstico, a dosagem da troponina I apresentou maior sensibilidade, retornado ao nível normal de 3 a 10 dias, detectando acertadamente a lesão cardíaca. Diante da gravidade da doença, é necessária maior divulgação dos sintomas, para que as pessoas possam procurar auxílio imediato.

Durante os últimos 25 anos isoformas cardíacos de troponina I e T têm sido amplamente utilizados para diagnóstico imunoquímicos de patologias associadas com a morte de cardiomiócitos (infarto do miocárdio, trauma do miocárdio, e outros).

O uso de testes de biomarcadores cardíacos na prática clínica depende da seleção adequada dos pacientes, da interpretação correta dos resultados dos testes e da incorporação dos testes de biomarcadores nos métodos de diagnóstico existentes.

REAS/EJCH | Vol. 11(13) | e940 | DOI: https://doi.org/10.25248/reas.e940.2019 Página 4 de 8 
LIMA OS e VISMARI

$\mathrm{L}$.

Diagnóstico; Marcadores Bioquímicos; Infarto

Infarto Agudo do Miocárdio; Biomarcadores

síndrome coronária aguda, enfarte agudo do miocárdio, marcadores cardíacos, troponinas

Biomarcadores farmacológicos; Infarto Agudo do Miocárdio

Doenças Cardiovasculares;

SBC

SBC

2014B

miocárdio

Marcadores Biológicos; Insuficiência

Cardíaca; Cardiomiopatias; Prática

Clínica Baseada em Evidências

Infarto Agudo do Miocárdio.

SOUZA LP, et al.

2016
Estudo de caso

O presente artigo sugere que no momento da admissão do paciente no serviço de emergência, haja a solicitação combinada da mioglobina e um marcador mais específico de necrose miocárdica (CK-MB) para a exclusão precoce de um infarto do miocárdio. Isto seria, além de eficaz para o diagnóstico, uma maneira mais viável financeiramente. Neste contexto, uma atenção especial deveria ser dada às mulheres atendidas com sintomas isquêmicos, tendo em vista a alta taxa de mortalidade a elas associada.

O Infarto Agudo do Miocárdio é responsável por uma alta porcentagem de mortes por doenças cardíacas, apresentando um grande problema de saúde pública. Com devida análise das dosagens dos marcadores séricos cardíacos, relacionados com o eletrocardiograma e presença de dor torácica, pode-se diagnosticar o Infarto Agudo do Miocárdio

Os biomarcadores podem fornecer, essencialmente, três tipos de informação nos doentes com síndrome coronária aguda. Primeiro, os biomarcadores podem ser úteis no diagnóstico imediato da Síndrome Coronariana Aguda

As troponinas T e I (na admissão e 6-9h após injúria) seguidas pela CK-MB massa são os biomarcadores sugeridos para avaliação de lesão miocárdica. O hs-Tnl e hs-TnT são muito sensíveis nos estágios precoces e a CK-MB massa tem sido útil para diagnóstico de reinfarto, pelo seu curto período de meia-vida

Revisão de

Literatura

Revisão de

Literatura

Biomarcador cardíaco é uma forma eficientes para o diagnóstico de doenças cardiovasculares

Existem diversos marcadores biológicos que podem auxiliar no diagnóstico de doenças cardiovasculares.

Os marcadores bioquímicos mais utilizados de imediato frente a um infarto agudo do miocárdio são: a mioglobina, troponina I e a fração CK-MB, existindo também outros tipos como Aspartatoaminotransferase (AST) e a Lactato desidrogenase (LDH).

REAS/EJCH | Vol. 11(13) | e940 | DOI: https://doi.org/10.25248/reas.e940.2019 Página 5 de 8 


\section{Revista Eletrônica Acervo Saúde / Electronic Journal Collection Health ～ISSN 2178-2091}

\begin{tabular}{|c|c|c|c|c|}
\hline CARDOSO MR, et al. & 2018 & $\begin{array}{l}\text { Síndrome Coronariana Aguda, } \\
\text { Troponina, Doenças Cardiovasculares / } \\
\text { Mortalidade, Infarto do Miocárdio, } \\
\text { Hospitalização / Economia }\end{array}$ & $\begin{array}{l}\text { Estudo } \\
\text { retrospectivo, } \\
\text { transversal com } \\
174 \text { indivíduos com } \\
\text { SCA }\end{array}$ & $\begin{array}{l}\text { Encontrada correlação linear positiva entre os níveis de TnUs e } \\
\text { complexidade das lesões coronarianas, assim como entre esse } \\
\text { biomarcador e os escores clínicos TIMl e GRACE }\end{array}$ \\
\hline
\end{tabular}

Fonte: Dados da pesquisa, 2019. 
A enzima Creatina-quinase (CK) é catalisadora do fosfato de adenosina e trifosfato (ATP) em fosfato de creatinina. Ela consiste na união de duas subunidades (B e $M)$, que são separadas de três formas diferentes. A primeira delas é CK-BB, que é dominante no cérebro e é raro estar presente no sangue. A segunda é CKMM, que é predominante no músculo esquelético e CK-MB, que é encontrada quase que exclusivamente no miocárdio (embora também seja encontrada em pequenas quantidades em outros tecidos). A concentração de CK- MB, nosso objeto de estudo, começa a ficar elevada de 3 a 8 horas depois da dor no coração, com pico alcançado em 24 horas (quando atinge o limite de seis a nove vezes de detecção) e normalizando em 48 a 72 horas (LOZOVOY MAB, et al., 2008; CANTELLE CF e LANARO R, 2011; SILVA SH e MORESCO RN, 2011; ROCHA K, 2012; BARBOSA L, 2014; BOUWMAN BBLM, 2014).

A atividade do CK-MB também pode se mostrar elevada em outras desordens cardíacas, como a desfibrilação, insuficiência coronária, fibrilação auricular crônica, ela é quase que exclusivamente produzida no miocárdio (LOZOVOY MAB, et al., 2008; CANTELLE CF e LANARO R, 2011; SILVA SH e MORESCO RN, 2011).

Embora seja um marcador tradicional, que apresente especificidade muito superior ao CK Total, a CK-MB possui limitações. De modo geral, a mesma deve ser medida por meio de imunoensaio para sua dosagem na sua concentração no plasma (CK-MB massa) em vez da sua atividade em si, isto porque a aferição realizada desta forma mostra maior sensibilidade e especificidade para IAM. Sua sensibilidade é de $50 \%$ após 3 horas do início dos sintomas e de $80 \%$ em seis horas. Sua principal limitação é a possibilidade de falso positivo, uma vez que este marcador também se mostra elevado após dano em outros tecidos que não são os cardíacos (SILVA SH e MORESCO RN, 2011; SBC, 2014; BOUWMAN BBLM, 2014).

De fato, esta enzima pode ser encontrada discretamente na corrente sanguínea de pessoas saudáveis, podendo ocorrer elevação de seus índices quando há também dano no músculo esquelético (SOUZA LP, et al, 2016). Apesar disso é de bastante utilidade para o diagnóstico do reinfarto, pois a CK-MB tem a meia-vida curta em comparação com a da troponina (MIRANDA MR e LIMA LM 2014).

A troponina é uma proteína reguladora da contração muscular, tanto de músculos estriados quanto cardíacos, localizada nos filamentos contrateis do miócito, mas que existem em pequena quantidade citosol (BOUWMAN BBLM, 2014).

Esta proteína pode ser dividida em três subunidades: C, I e T. A primeira delas (troponina C) se liga ao cálcio e é coexpressa nas fibras musculares esqueléticas e, por isso mesmo, não é um marcador cardíaco específico, como é o caso das Troponinas I (Tnlc) e Troponinas T (TnTc), que tem uma estrutura genética e proteica definida por sequências de aminoácidos específica que permite o diagnóstico do IAM. Estas proteínas possuem funções bem específicas. A troponina I se liga a actina e inibe a interação actina-miosina, prevenindo na ausência do cálcio. A troponina $T$, se liga diretamente a tropomiosina (LOZOVOY MAB, et al., 2008; CANTELLE CF e LANARO R, 2011; KATRUKHA IA, 2013; SBC, 2014; BARBOSA L, 2014; BOUWMAN BBLM, 2014).

Alguns ensaios com troponinas cardíacas ( $\mathrm{TnT}$ e $\mathrm{Tnl}$ ) tem apresentado vantagens em comparação com os realizados com CK-MB massa, sendo essas: maior especificidade para lesão cardíaca, uma vez que a CKMB pode ser encontrada em tecidos que não sejam do coração; e, a habilidade de detectar pequenas quantidades de lesão miocárdica, o que não é detectável pela enzima CK-MB (SBC, 2014). Em razão de sua alta especificidade e sensibilidade estas proteínas são consideradas marcadores ouro para o diagnóstico de IAM (SILVA SH e MORESCO RN, 2011).

A sensibilidade destas troponinas varia de acordo com o tempo, mas a especificidade não, mantendo-se com valores aproximados de 83\% a 98\% (LIMA OS e VISMARI L, 2014; BARBOSA L, 2014)

De modo geral, as troponinas apresentam a vantagem de manter níveis altos por um período prolongado de aproximadamente de 24 horas (quando alcança até mais de 40 vezes o limite de detecção) depois do início dos sinais clínicos de IAM observando-se que sua liberação começa de 4 a 8 horas depois dos sintomas iniciais (ROCHA K, 2012; LIMA OS e VISMARI L, 2014; SOUZA LP, et al., 2016). 


\section{CONCLUSÃO}

Entre os marcadores cardíacos atualmente utilizados, os mais solicitados pelos profissionais da saúde são as troponinas, Isoenzima CK-MB e a mioglobina. Apresentadas as funções, e levando-se em consideração aspectos como especificidade, sensibilidade e perfil de liberação na corrente sanguínea, concluiu-se que a Mioglobina e a fração MB da Creatinoquinase (CK-MB) possuem baixa especificidade por estarem presentes tanto na musculatura esquelética como também na musculatura cardíaca, mas são considerados sensíveis. Diferentemente das Troponinas cardíacas $\mathrm{T}$ e I, que mostram ter maior especificidade para o coração e sensibilidade clínica alta. E em caso de reinfarto o biomarcador cardíaco mais especifico é o CK-MB massa, pois sua meia vida é mais curta comparada com a Troponina, facilitando assim o diagnóstico.

\section{AGRADECIMENTOS E FINANCIAMENTO}

Agradecemos ao nosso orientador Prof. Dr. Lysandro Pinto Borges por toda dedicação e apoio ao nosso trabalho. Ao LaBiC (Laboratório de Bioquímica Clínica da Universidade Federal de Sergipe) e gostaríamos também de agradecer a nossa Instituição de ensino, Universidade Federal de Sergipe por possibilitar a realização deste trabalho científico.

\section{REFERÊNCIAS}

1. BARBOSA L. Os marcadores bioquímicos no diagnóstico do infarto agudo do miocárdio. Dissertação (Iniciação em Biomedicina). Unicid (Universidade da Cidade de São Paulo), São Paulo, 2014.

2. Biomarcadores em Cardiologia - Parte 1 - Na Insuficiência Cardíaca e nas Cardiomiopatias Específicas. Arq. Bras. Cardio. I. 2014; 103(6):451-45 (2014b).

3. BOUWMAN BBLM. Biomarcadores cardíacos no diagnóstico da Síndrome Coronária Aguda. (Dissertação de Mestrado). Ciências Farmacêuticas. Universidade do Algarve, 2014.

4. CANTELLE CF, LANARO R. Indicadores Bioquímicos do Infarto Agudo do Miocárdio. Revista Ciências em Saúde v1, $\mathrm{n} 3$ out 2011.

5. CARDOSO MR; SILVA JUNIOR, DG; RIBEIRO, EA; ROCHA NETO, AM. Correlação entre a Complexidade das Lesões Coronarianas e os Níveis de Troponina Ultrassensível em Pacientes com Síndrome Coronariana Aguda. International Journal of Cardiovascular Sciences. 2018;31(3)218-225.

6. KATRUKHA IA. Complexo troponina cardíaca humana. Estrutura e funções. Bioquímica (Moscow), 2013, Vol. 78, No. 13, pp. 1447-1465.

7. LIMA OS, VISMARI L. Avaliação dos marcadores de lesão miocárdica solicitados em hospital paulista. Infarma Ciências Farmacêuticas. v26.e3. a2014. pp166-171.

8. LOZOVOY MAB, PRIESNITZ JC, SILVA AS. Infarto Agudo do Miocárdio: aspectos clínicos e laboratoriais. Interbio V.2 n. $1 ; 2008$.

9. MARTINS CS. Troponina: estrutura, fisiopatologia e importância Clínica para Além da Isquemia Miocárdica. Arquivos de medicina. Vol. 23, № 6 23(6):221-40, 2009.

1. 10, MIRANDA MR, LIMA LM. Marcadores bioquímicos do infarto agudo do miocárdio. Rev Med. Minas Gerais 2014; 24(1): 98-10598.

10. OYAMA MA. Using cardiac biomarkers in veterinary practice. Vet Clin Small Anim. In press 2013.

11. ROCHA K. Marcadores bioquímicos de lesão no miocárdio. 10ª Mostra acadêmica UNIMEP, 2012.

12. SILVA SH, MORESCO RN. Biomarcadores cardíacos na avaliação da síndrome coronariana aguda. Scientia Medica (Porto Alegre) 2011; volume 21, número 3, p. 132-142.

13. SOCIEDADE BRASILEIRA DE CARDIOLOGIA (SBC). Arquivos Brasileiros de Cardiologia. Volume 102, № 3, Suplemento 1, março:2014.

14. SOUZA LP; MARTINS, WS; GONÇALVES, JS; MACHADO, MCFP; RODRIGUES, PMB. Marcadores bioquímicos no infarto agudo do miocárdio: revisão de literatura. Saúde. Volume 16, Número 3, João Pessoa, 2016. 